357 THE TWO-PHASE DESIGN MAKES EFFICIENT USE OF EXPERT-TIME IN ASSESSING EXPOSURE TO OCCUPATIONAL CARCINOGENS: A LUNG CANCER CASECONTROL STUDY

P Wild. INRS, Vandoeuvre Les Nancy Cedex, France

10.1136/oemed-2013-101717.357

Objectives To identify dose-response relationship between lung cancer incidence and cumulative exposure to the main occupational carcinogens as assessed by experts making use of an initial algorithmic exposure assessment within the two-phase design.

Methods A population-based case-control study including 246 cases and 531 controls was conducted in North-Eastern France. Detailed occupational and personal risk factors were obtained in face-to-face interviews based on a task-based questionnaire and a series of job-specific questionnaires. In the conceptual framework of a two-phase design, cumulative expert-based exposure scores were obtained in a subset of 215 cases and 269 controls stratified on smoking and a prior algorithmic exposure assessment. The cases and controls included in the expert assessment were chosen in order to over-sample rare exposure categories. The data were analysed using logistic regression models adapted to two-phase data. This analysis makes use of the subset of subjects with expert assessments but also of the initial data set from which the subset was sampled.

Results Expert based cumulative exposure scores were lower than the corresponding algorithmic scores. The correlation between algorithmic and expert-assessed cumulative exposure scores were high for asbestos $(\mathrm{r}=0.80)$ but less so for crystalline silica $(\mathrm{CS})(\mathrm{r}=0.60)$ and polycyclic aromatic hydrocarbons -PAH $(r=0.56)$. We identified significant dose-response relationships (DRR) for asbestos, CS and Diesel Motor Exhaust (DME) with significant ORs exceeding 2 in the respective highest tertiles of cumulative exposure. The dose-response relationship with $\mathrm{PAH}$ was borderline significant but the OR in the highest tertile was 1.96 (95\%CI [1.11-3.46]). All DRRs were steeper when using expert-based scores than when using algorithmic scores.

Conclusion Dose-response relationships between lung cancer incidence and cumulative exposure scores could be identified based on expert-assessment in a subset of cases and controls chosen to be informative in the framework of a two-phase design.

\section{UNDERSTANDING FACTORS RELATED TO BETWEEN- AND WITHIN-SUBJECT VARIATION IN AN EFFORT TO BETTER DEFINE SEGS}

${ }^{1}$ P J D Dopart, 'Dalton, ${ }^{2}$ Maute, 'Lees. 'Johns Hopkins University, Baltimore, United States of America; ${ }^{2}$ Monell Chemical Senses Center, Philadelphia, United States of America

\subsection{6/oemed-2013-101717.358}

Objectives One approach for characterising exposures of an occupational cohort when measurement data are limited is to divide the population into Similar Exposure Groups (SEGs), or clusters of workers believed to have the same general exposure profile for the agent (s) under study, from which individual levels are established. However, when the assumed homogeneity of an SEG is incorrect, researchers risk misclassifying exposures. Through analyses of formaldehyde exposures of veterinary students enrolled in a gross anatomy laboratory course, this research examines methods for improved understanding of variability sources within a dataset in an effort to better define SEGs.
Methods Initial analyses suggested classifying this cohort as one single SEG may be questionable and demonstrated the importance of an appropriate sampling strategy. A mixed-effects model was thus used to identify exposure determinants and assess sources of variation. Using formaldehyde exposure as the dependent variable, explanatory variables were partitioned into fixed effects (animal, animal part, lab location, sample collection date) and random effects (subject). Additional analyses were run separately for each animal type in an effort to examine variability by task. Results Examination of the data identified several potential sources of variability. The model indicated that animal and animal part may have a significant effect on exposure, with a withinsubject to between-subject variance ratio of $2: 1$. The proportion of total variability attributable to within-subject variation differed by animal type, with $46.4,98.6$, and $70.2 \%$ associated with dog, goat, and horse dissections, respectively.

Conclusions These results help identify and describe work characteristics influencing exposure levels within the cohort. Understanding factors related to between- and within-subject variability allows for refined sub-grouping of the population and identification of work conditions that influence day-to-day exposure variations. With on-going analyses, this work will attempt to create more informative SEGs as a way to reduce exposure misclassification.

\section{AN EXPOSURE ASSESSMENT MODEL FOR INADVERTENT INGESTION EXPOSURE}

${ }^{1} \mathrm{M}$ G N Gorman Ng, 'van Tongeren, ${ }^{2}$ Semple. 'Institute of Occupational Medicine, Edinburgh, United Kingdom; ${ }^{2}$ University of Aberdeen, Aberdeen, United Kingdom

\subsection{6/oemed-2013-101717.359}

Inadvertent ingestion exposure (IIE) arises from contact between the mouth and contaminated hands and objects. It may be a significant route of occupational exposure for metals, pharmaceuticals, pesticides and infectious agents (Cherrie et al, 2006). We have developed a predictive model of IIE. The deterministic model structure was based on data collected in: (i) a literature review of the determinants of ingestion exposure; (ii) a database of published transfer efficiencies for dermal and oral transfer; (iii) laboratory experiments to determine the efficiency of transfer of materials between hands, glove, objects and the mouth in different scenarios; and (iv) real-time observations of workers to identify rates and determinants of handto-mouth behaviour. The model estimates exposure to the hands and the perioral area (the area around the mouth). It was validated against measurements of hand and perioral exposure taken from 65 exposure scenarios (exposure to metals, pesticides, and cytotoxic drugs) across 13 worksites. Using written descriptions of worksites and practices, three exposure assessors, blinded to the measurement data, generated model estimates that were compared to the measured data. The correlation coefficient between model estimates and measurements ranged from $0.69-0.81$ for hands and from $0.74-0.84$ for perioral. The model was relatively reliable between assessors with between-assessor correlation coefficients ranging from $0.75-0.98$. The model was designed to be used to estimate exposure at the job group level and could be used to develop job exposure matrices. Because the model can be used with historical written descriptions of tasks it may also be used to create retrospective exposure estimates. Based on the validation that has been conducted, the model appears to be a promising 